\section{Trauma Surgery} \& Acute Care Open

\title{
Complex chest wall injury and repair complicated by empyema
}

\author{
Lukas Mueller, ${ }^{1}$ Cecilia Benz, ${ }^{1}$ Steven Briggs, ${ }^{2}$ Cornelius Dyke ${ }^{3}$
}

${ }^{1}$ General Surgery, University of North Dakota School of Medicine and Health Sciences, Grand Forks, North Dakota, USA 2Trauma, Sanford Medical Center Fargo, Fargo, North Dakota, USA

${ }^{3}$ Surgery, University of North Dakota School of Medicine and Health Sciences, Fargo, North Dakota, USA

Correspondence to Dr Lukas Mueller; lukas. mueller@ndus.edu (c) Author(s) (or their employer(s)) 2020. Re-use permitted under CC BY-NC. No commercial re-use. See rights and permissions. Published by BMJ.

To cite: Mueller $L$, Benz C, Briggs $S$, et al. Trauma Surg Acute Care Open

2020:5:e000395.

\section{CASE SUMMARY}

A 24-year-old man presented to the emergency department after blunt thoracic trauma. As a vested rider, he was competing in a rodeo bull riding competition when he was thrown and suffered a bull stomp to his left thoracic cage. At presentation, he was hemodynamically stable with an obviously deformed left anterolateral chest wall. Complete trauma evaluation was performed and injury was limited to the chest with fractures of ribs 1-8 and a moderate left pleural effusion. Ribs 3-7 were comminuted and severely displaced (figure 1). Severe pulmonary contusion was present. He underwent left tube thoracostomy with evacuation of the bloody effusion. The patient did not require mechanical ventilation and remained clinically stable.

The patient was stabilized in the surgical intensive care unit and on hospital day 3 underwent left thoracoscopy, left lateral thoracotomy, wedge resection of the lung, and stabilization of left rib fractures with plating due to significant rib displacement, persistent pain, and splinting with inspiration. The displaced fracture line ran obliquely along the mid to posterior axillary line from the third to seventh ribs with significant displacement of the posterior segments into the left upper lobe parenchyma near the hilum. No active bleeding was present. The left upper lobe laceration was resected in a non-anatomic sublobar fashion using a stapler. Open reduction and internal fixation of ribs 3-7 with titanium plate and screw rib fixation device was accomplished (figure 2). Intercostal muscle tissue loss was extensive and covered with the latissimus dorsi muscle. Chest tubes were placed and the incision was closed anatomically.

Postoperatively, the patient was extubated and remained hemodynamically stable. On postoperative day 10 , he developed a productive cough, fever, and leukocytosis. Repeat chest CT demonstrated a left-sided pneumonia and left pleural effusion consistent with empyema (figure 3).

\section{WHAT WOULD YOU DO?}

Non-operative management with antibiotics alone? Non-operative management with percutaneous drainage?

Video-assisted thoracoscopic surgery (VATS)/thoracotomy with drainage?

VATS/thoracotomy with drainage and removal of hardware?

\section{WHAT WE DID AND WHY}

Antibiotic coverage was initiated. We performed a combined thoracotomy and thoracoscopy

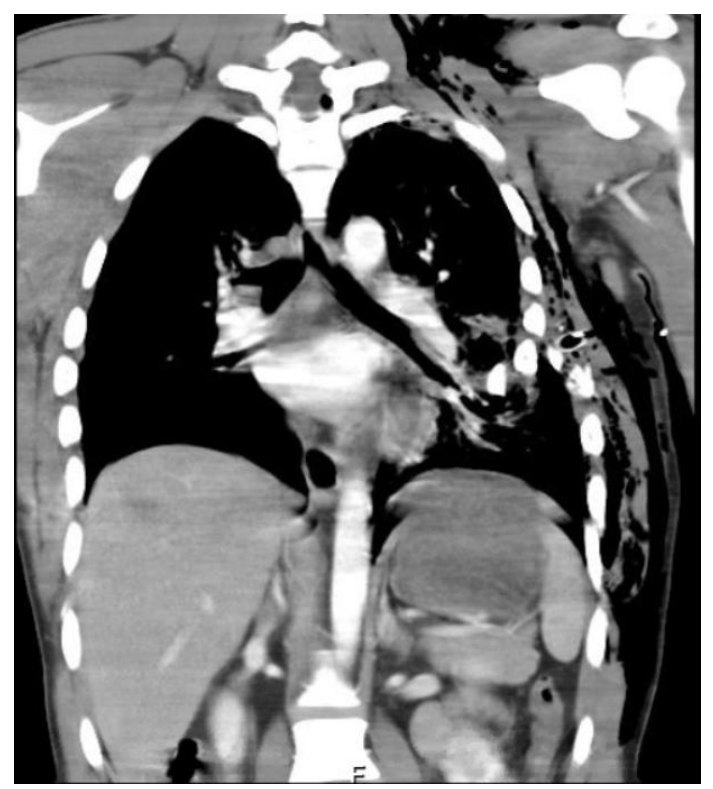

Figure 1 Preoperative CT. Imaging demonstrates significant chest wall injury of severely displaced left ribs with concern of parenchymal violation.

approach with operative drainage of the left chest without hardware removal. The previous incision was partially opened posteriorly and a combined approach was performed to minimize the thoracotomy incision and to assist in dissection and drainage of the loculated effusion. No subcutaneous or chest wall abscess was appreciated. Consideration for rib plate removal was given; however, the decision was made to maintain the titanium rib plates in the absence of gross contamination and because of the complexity of the injury. Normal saline and

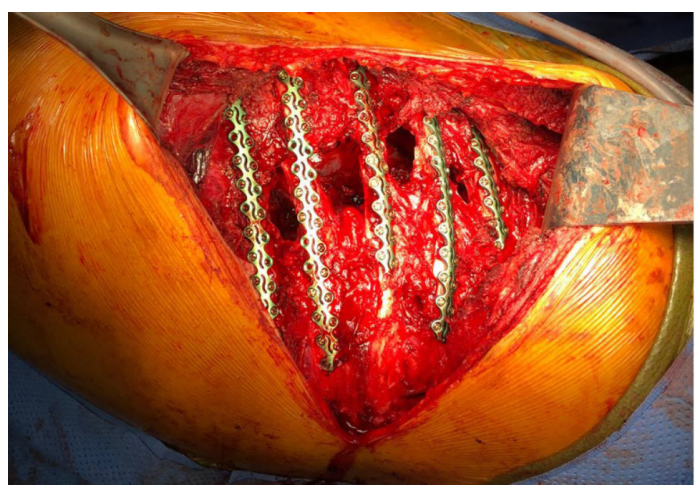

Figure 2 Intraoperative fixation. Image exhibits the significant muscle tissue loss secondary to traumatic injury and the subsequent titanium rib plating repair. Ribs plated 3-7. 


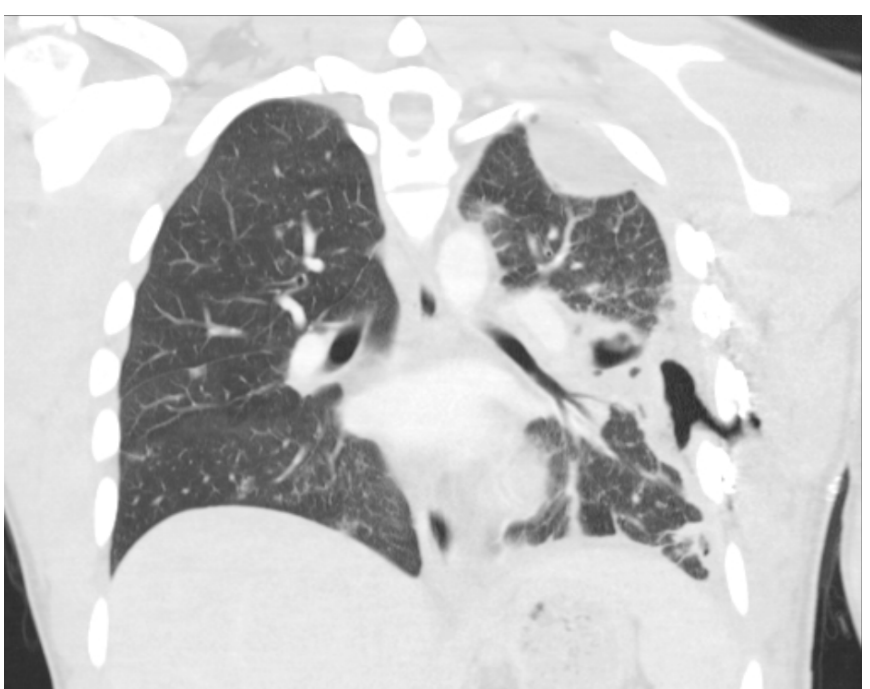

Figure 3 Postoperative CT. Imaging demonstrates reconstruction of the displaced ribs. More importantly, depicted is the postoperative development of an empyema with features concerning for an entrapped lung.

antibiotic lavage of the pleural space was done and drained with two large-bore chest tubes. The subcutaneous layer was allowed to heal by secondary intention.

After re-exploration, the patient recovered well. Pleural space cultures resulted in Serratia marcescens without bacteremia. Due to the rodeo environment exposure, polymicrobial intravenous antibiotic coverage was continued and transitioned to tailored oral therapy for 4 weeks. He was discharged on postoperative day 16 from the initial procedure to his home state with follow-up care coordinated with infectious disease, wound care, and primary care. Three-month and 16-month phone follow-up revealed the patient was asymptomatic, his wound well healed, and no thoracic wall or pulmonary issues. He elected to have no further surgery for hardware removal. Despite being strongly advised to refrain from bull riding, he has resumed his bullriding career without any setbacks.

In our opinion, the patient required surgical drainage of his empyema. We do acknowledge an alternative strategy of drainage through a separate incision without exposure of the rib plates. Removal of the hardware at the time of drainage was discussed extensively. The decision to leave the rib plates intact was based on no gross contamination of the rib plates, the inertness of the titanium hardware system, the quality of coverage with the viable latissimus dorsi muscle, and the underlying extensive rib and chest wall injury. Isolated reports of rib fixation in a prior infected field do exist; however, the reports, and together with this report, suggest a relatively low risk of hardware infection, although experience is limited and there is no consensus. In recommendation, it appears safe to proceed with drainage and prolonged antibiotic coverage without removal of rib plating hardware in situations where rib plating removal would result in thoracic cage collapse.

Contributors All authors contributed equally to the drafting of this article and accept responsibility.

Funding The authors have not declared a specific grant for this research from any funding agency in the public, commercial or not-for-profit sectors.

Competing interests None declared.

Patient consent for publication Not required.

Ethics approval Local institutional review board approval was not obtained as this case report was not deemed to be clinical research.

Provenance and peer review Not commissioned; externally peer reviewed.

Open access This is an open access article distributed in accordance with the Creative Commons Attribution Non Commercial (CC BY-NC 4.0) license, which permits others to distribute, remix, adapt, build upon this work non-commercially, and license their derivative works on different terms, provided the original work is properly cited, appropriate credit is given, any changes made indicated, and the use is non-commercial. See: http://creativecommons.org/licenses/by-nc/4.0/. 